\title{
Tumor necrosis factor receptor-associated factor 6 promotes migration of rheumatoid arthritis fibroblast-like synoviocytes
}

\author{
HUIQIN WANG ${ }^{1}$, WEIXIA CHEN ${ }^{1}$, LING WANG ${ }^{2}$, FAXIN LI $^{3}$, CHUNLING ZHANG $^{3}$ and LI XU ${ }^{1}$ \\ Departments of ${ }^{1}$ Clinical Laboratory and ${ }^{2}$ Rheumatology, Zaozhuang Municipal Hospital, Zaozhuang, Shandong 277102; \\ ${ }^{3}$ Department of Rheumatology, Jinan Central Hospital Affiliated to Shandong University, Jinan, Shandong 250204, P.R. China
}

Received February 5, 2014; Accepted November 3, 2014

DOI: $10.3892 / \mathrm{mmr} .2014 .3104$

\begin{abstract}
Fibroblast-like synoviocytes (FLSs) have a pivotal role in the destruction of joints in rheumatoid arthritis (RA). Tumor necrosis factor receptor-associated factor 6 (TRAF6) is a critical mediator in the inflammatory pathway and of the activity of osteoclasts. The aim of the present study was to investigate whether TRAF6 is involved in the progression of RA in mouse collagen-induced arthritis (CIA) and human RA FLSs in vitro. In vivo mouse models were transfected with TRAF6 small interfering (si)RNA (siTRAF6) and TRAF6 inhibition was achieved in FLSs using an anti-TRAF6 monoclonal antibody in vitro in order to assess the effects of TRAF6 inhibition on the migration and invasion of FLSs. Inhibition of TRAF6 using mouse specific siTRAF6 reduced the severity of arthritis and joint inflammation. Serum anti-collagen II antibodies, matrix metalloproteinase (MMP)-1, MMP-3 and MMP-9 were also inhibited in CIA mice by siTRAF6. The levels of MMPs produced by IL-1 $\beta$-stimulated human RA-FLSs were reduced by anti-TRAF6 monoclonal antibody. TRAF6 blockade significantly suppressed the IL- $1 \beta$-stimulated migration and invasion of human RA-FLSs. These results support a role for TRAF6 in the pathogenesis of RA, and suggest that the TRAF6 blockade may be a potential strategy in the management of RA.
\end{abstract}

\section{Introduction}

Rheumatoid arthritis (RA) is a synovial disease characterized by chronic inflammation of the joints culminating in joint destruction. Proliferative fibroblast-like synoviocytes (FLSs) have crucial roles in joint inflammation and bone damage, as they produce large quantities of pro-inflammatory mediators,

Correspondence to: Dr Li Xu, Department of Clinical Laboratory, Zaozhuang Municipal Hospital, 41 Longtou Road, Zaozhuang, Shandong 277102, P.R. China

E-mail: zaozhuangxuli@yeah.net

Key words: rheumatoid arthritis, collagen-induced arthritis, tumor necrosis factor receptor-associated factor 6, fibroblast-like synoviocytes including interleukin (IL)-1, IL-6, tumor necrosis factor- $\alpha$ (TNF- $\alpha$ ), matrix metalloproteinases (MMPs) and prostaglandin E2 (1). These mediators bind to specific receptors, causing gene transcription, and form complicated signaling interactions that contribute to the progression of inflammatory arthritis, including leukocyte infiltration, cytokine network formation and cartilage catabolism elevation (2). The manner in which rheumatoid arthritis is treated has changed markedly with the introduction of anti-tumor necrosis factor (anti-TNF) biologics; however, a number of patients still have less than adequate control of their disease, even with these therapeutic regimens involving disease-modifying anti-rheumatic drugs and currently available biologics (3). Thus, there is a requirement for safer and more effective anti-arthritic strategies for long-term use.

Tumor necrosis factor receptor (TNFR)-associated factor (TRAF) 6 transduces signals from several members of the TNFR superfamily and the TLR/IL-1R family to activate the transcription factors nuclear factor $(\mathrm{NF})-\kappa \mathrm{B}$ and activator protein (AP)-1 (4). It has been also shown that TRAF6 is required for NF- $\kappa$ B activation (5). TRAF6 has been shown to be critically involved in regulating inflammatory response signaling pathways involving adaptive immunity, innate immunity and bone metabolism (6). As TRAF6 regulates inflammation and links immunity to bone metabolism, the aim of the current study was to explore the role of TRAF6 in the development of the collagen-induced arthritis (CIA) model and the migration of human RA-FLSs.

\section{Materials and methods}

Mouse collagen-induced arthritis and evaluation. Sixteen 10-week-old male DBA/1 mice were purchased from the Chinese Academy of Sciences (Shanghai, China) and were maintained under a controlled temperature $\left(22^{\circ} \mathrm{C}\right)$ and a 12-h light/dark cycle (light, 0700-1900 h), with access to a water and a standard diet ad libitum. Mice were immunized with Freund's adjuvant (Sigma-Aldrich, St. Louis, MO, USA)) and $100 \mu \mathrm{g}$ bovine type II collagen (Chondrex, Redmond, WA, USA) at the base of the tail on day 0 and day 21 as previously described (7,8). Animal experimental protocols were approved by the Animal Committee of Zaozhuang Municipal Hospital (Zaozhuang, China). Clinical arthritic scoring was performed every three days as follows (9): 0 , normal; 1 , mild; 2 , moderate; 
and 3, maximal redness and swelling. The maximum score per paw was 3 with a total maximum score of 12 per mouse.

TRAF6 inhibition in vivo and in vitro. Small interfering RNA (siRNA) against TRAF6 (siTRAF6) and control siRNA were obtained from Santa Cruz Biotechnology Inc. (Santa Cruz, CA, USA). The siTRAF6 was delivered in vivo using in vivo-jet polyethylene imine (PEI, Polyplus-transfection, Qbiogene, CA, USA) according to the manufacturer's instructions. In brief, siTRAF6 and PEI were mixed and dissolved in $400 \mu \mathrm{l} 5 \%$ glucose for $20 \mathrm{~min}$ for intraperitoneal injection at room temperature, and the mixture was administered three times daily, three days a week over three weeks. Mixtures containing control siRNA was used as control.

In vitro, TRAF6 inhibition was achieved with an anti-TRAF6 monoclonal antibody (anti-TRAF6mAb; Santa Cruz Biotechnology, Inc.). FLSs were seeded in 6-well plates at $1 \times 10^{6}$ cells/well.

Histopathology. The mouse hind limbs were fixed in $4 \%$ paraformaldehyde, decalcified and embedded in paraffin. Serial 4- $\mu \mathrm{m}$ sections were cut and stained with hematoxylin and eosin (H\&E). Sections were analyzed microscopically for the extent of inflammation and bone erosion as previously reported (10). The histological scores were determined as follows: Score 0 , no signs of inflammation; 1 , mild inflammation without cartilage destruction; 2-4, increasing degrees of inflammatory cell infiltration and cartilage/bone destruction.

Human RA patients. Twenty newly diagnosed active RA patients, who had not received any treatment, who fulfilled the 2010 American College of Rheumatology/European League Against Rheumatism criteria for RA (11), were recruited from the Zaozhuang Municipal Hospital (Zaozhuang, China). Active disease was defined as a Disease Activity Score 28-joint assessment (DAS28) of $>5.1$ (12). This study was performed with the approval of the Ethics Committee of Zaozhuang Municipal Hospital (Zaozhuang, China) in accordance with the Helsinki Declaration. All patients provided informed consent.

Cell isolation and culture. RA-FLSs were obtained from the synovium during knee joint arthroscopy. FLSs were isolated from synovial tissues by enzymatic digestion as previously described (13). RA-FLSs were grown in Dulbecco's modified Eagle's medium (DMEM; Thermo Fisher Scientific, Waltham, MA, USA) containing $10 \%$ fetal bovine serum (Thermo Fisher Scientific) in a humidified incubator at $37^{\circ} \mathrm{C}$.

Western blot analysis. Total protein was extracted from the joints and FLSs using an radioimmunoprecipitation assay buffer (Sigma-Aldrich). The protein concentration was measured with a DC Protein assay (Bio-Rad, Hercules, CA, USA). Proteins were separated using $10 \%$ SDS-PAGE, and then transferred to a nitrocellulose membrane (Millipore, Billerica, MA, USA). After blocking in 5\% skimmed milk, the membranes were incubated with rabbit polyclonal anti-TRAF6 antibody (1:1,000; sc-7221; Santa Cruz Biotechnology, Inc.) or rabbit polyclonal anti-GAPDH (internal control; 1:2,000; sc-25778; Santa Cruz Biotechnology, Inc.) overnight. Thereafter, the membranes were rinsed in blocking solution and incubated for $1 \mathrm{~h}$ with a secondary mouse anti-rabbit antibody conjugated to horseradish peroxidase (1:2,000; sc-53804; Santa Cruz Biotechnology, Inc.). Bands were visualized using an acridan-based substrate detection system (ECL; Millipore).

ELISA. At the end of experiment, animals were sacrificed under $40 \mathrm{mg} / \mathrm{kg}$ body weight pentobarbitol administered intraperitoneally anesthetic and sera were obtainedby cardiac puncture. The anti-collagen II antibodies (anti-CII)ELISAs (Chondrex, USA) and MMP-1, MMP-3 and MMP-9 ELISAs (R\&D Systems) were performed according to the manufacturer's instructions.

Determination of MMP-1, MMP-3 and MMP-9 in the supernatants. Cells were seeded at a density of $1 \times 10^{6} / \mathrm{ml}$ and pretreated with anti-TRAF6mAb for $24 \mathrm{~h}$, followed by a 72 -h stimulation with human IL-1 $\beta$ (50 ng/ml, eBioscience, San Diego, CA, USA). Cell supernatants were centrifuged at 1,500 x $\mathrm{g}$ for $10 \mathrm{~min}$ to avoid any cell debris prior to ELISA analysis. The levels of MMP-1, MMP-3 and MMP-9 were determined by Human MMP-3 and MMP-9 Quantikine ELISA kits (R\&D Systems, Shanghai, China) according to the manufacturer's instructions.

Cell migration and invasion. FLSs were seeded at a density of 4,000 cells/well in a 96-well plate. After treatment with the anti-TRAF6mAb and IL-1 $\beta$, the cells were observed under a ECLIPSE 80i fluorescent microscope (Nikon, Melville, NJ, USA). In order to assay cell migration, cells were seeded on 24-well plates coated with diluted gelatin (Sigma-Aldrich) at a density of $5 \times 10^{5}$. Once the cells reached $80 \%$ confluence, they were wounded by dragging a plastic pipette tip across the cell monolayer. Cells were then incubated in DMEM with $1 \%$ serum. Five fields of vision were selected at random and the migrated distances of cells were measured under a CarlZeiss LSM710 light microscope (Carl Zeiss, Oberkochen, Germany). Experiments were repeated a minimum of three times. Wounded monolayers were washed with phosphate-buffered saline (Thermo Fisher Scientific) to remove detached cells. The ability of FLSs to close the wounded space was used to assess their migration ability. Transwell migration assays were performed using a 24-well Boyden chamber $(8.0 \mu \mathrm{m}$; BD Biosciences, Franklin Lakes, NJ, USA) according to the manufacturer's instructions. In brief, FLSs were treated with anti-TRAF6mAb and cultured for $24 \mathrm{~h}$. Cells were then harvested by trypsinization, washed and resuspended in serum-free media at a density of $2 \times 10^{4}$ cells/well. A $100 \mu \mathrm{l}$ cell suspension was placed onto the upper and the lower chambers of the Transwell, which were filled with $500 \mu 1$ media containing serum as an adhesive substrate. Cells were incubated at $37^{\circ} \mathrm{C}$ under $5 \% \mathrm{CO}_{2}$ for $24 \mathrm{~h}$ and then non-migrating cells on the upper side of the membrane were removed with a cotton swab. Migrating cells on the lower side of the membrane were fixed with $4 \%$ methanol for $20 \mathrm{~min}$ and stained with $0.1 \%$ crystal violet for $1 \mathrm{~min}$. Photomicrographs (Carl Zeiss) of five random fields were obtained, and cells were counted to calculate the average number of cells that migrated. For the in vitro invasion assay, similar experiments were performed using inserts coated with a Matrigel baSDent membrane matrix (Thermo Fisher Scientific). The Matrigel (BD Biosciences) was diluted 


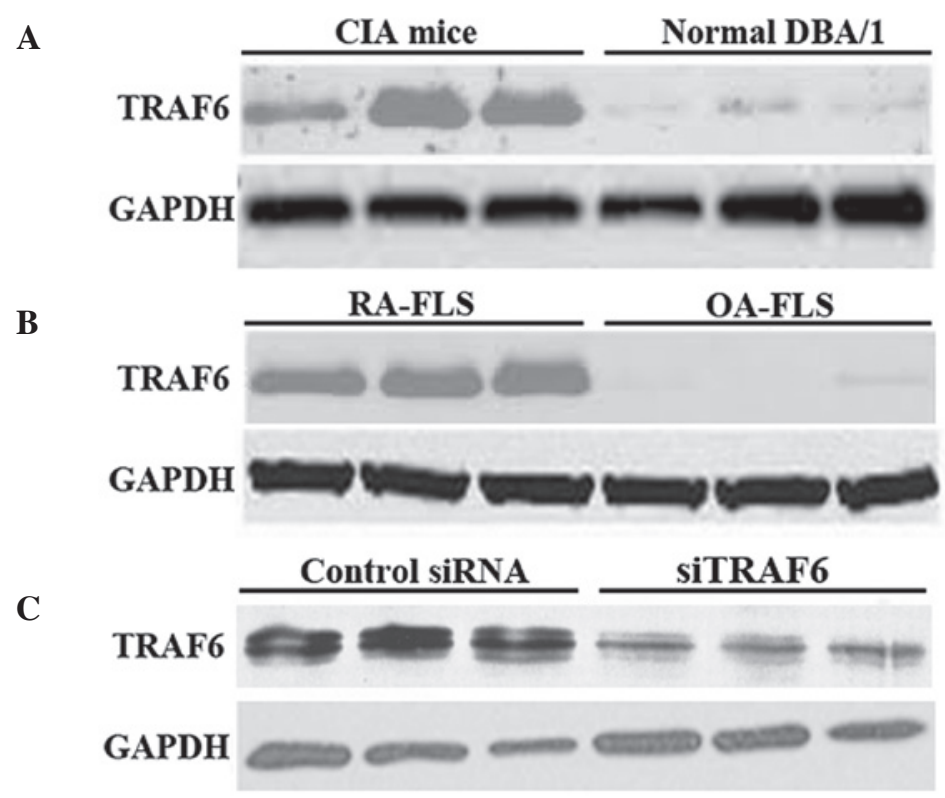

Figure 1. Upregulated TRAF6 in collagen-induced arthritis (CIA) mice and human rheumatoid arthritis fibroblast-like synoviocytes (RA-FLSs). (A) CIA mice vs normal DBA/1 mice. (B) Human RA-FLSs vs. osteoarthritis (OA)-FLSs. (C) Control siRNA-treated CIA mice vs. siTRAF6-treated CIA mice. In vivo siTRAF6 knocked down TRAF6 expression in CIA joints. The images are representative of three independent experiments.

A


Figure 2. In vivo siTRAF6 alleviated the collagen-induced arthritis (CIA). Arthritis score was recorded every $72 \mathrm{~h}$. Treatment with siTRAF6 was initiated on day 27 after the first immunization, when arthritis occurred in all mice. (A) Arthritis score during the treatment. (B) Mouse CIA joints stained with hematoxylin and eosin (scale bar, $100 \mu \mathrm{m}$ ). (C) Semiquantitative histological analysis ${ }^{*} \mathrm{P}<0.01$ vs. control siRNA-treated CIA mice; $\mathrm{n}=8 \mathrm{mice}$ per group.

in serum-free cold media and placed into upper chambers of a 24-well Transwell plate and incubated at $37^{\circ} \mathrm{C}$ for $1 \mathrm{~h}$. Cells were resuspended with serum-free media at a density of $5 \times 10^{4}$ cells/well and incubated for $48 \mathrm{~h}$ to evaluate cell migration.

Statistical analyses. All data are expressed as the mean \pm standard deviation. An unpaired t-test (two-tailed) was applied. Statistical analyses were performed using the SPSS software version 17.0 (SPSS, Inc., Chicago, IL, USA). P $<0.05$ was considered to indicate a statistically significant difference.

\section{Results}

TRAF6 expression is elevated in CIA joints and human RA-FLSs. The expression level of TRAF6 was detected by western blot analysis. The expression level of TRAF6 was 
A
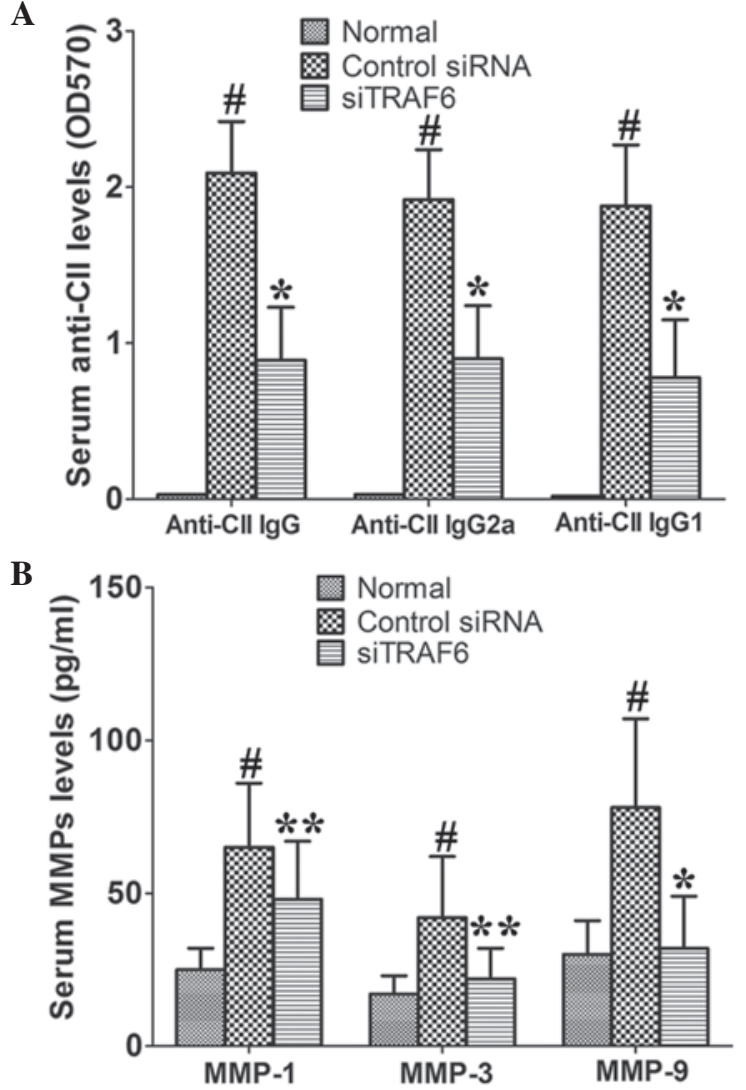

Figure 3. Serum anti-collagen II antibodies (anti-CII), matrix metalloproteinase (MMP)-1, MMP-3 and MMP-9 were reduced by siTRAF6 in collagen-induced arthritis (CIA) mice. (A) Serum levels of anti-CII. (B) Serum levels of MMP-1, MMP-3 and MMP-9. " $\mathrm{P}<0.01$, vs. normal DBA $/ 1$ mice; ${ }^{*} \mathrm{P}<0.01,{ }^{* *} \mathrm{P}<0.05$ vs. control siRNA-treated CIA mice $n=8$ mice per group.

upregulated in the joints of CIA mice compared with that of the normal DBA/1 mice (Fig. 1A). Consistently, the protein expression of TRAF6 was upregulated in human RA-FLSs (Fig. 1B). Furthermore, western blot analysis confirmed that TRAF6 was knocked down in CIA mice by in vivo siTRAF6 (Fig. 1C). These results suggest that aberrant TRAF6 expression may participate in the pathogenesis of RA.

TRAF6 knockdown reduces arthritis and histological damage in CIA mice. Treatment with siTRAF6 started on day 27 after first immunization, when arthritis was present in all mice. TRAF6 knockdown reduced the arthritis score of CIA mice (Fig. 2A). Consistently, the results showed that the histopathological damage, including inflammatory cell infiltration and bone destruction, was significantly attenuated in siTRAF6-treated CIA mice compared with that of the control siRNA-treated CIA mice (Fig. 2B and C).

Serum anti-CII antibodies and MMPs are reduced by siTRAF6 in CIA mice. In vivo TRAF6 knockdown significantly reduced serum anti-CII IgG, IgG1 and IgG2a as compared with those of the control siRNA-treated CIA mice (Fig. 3A). In addition, the data showed that siTRAF6 inhibited the production of serum MMP-1, MMP-3 and MMP-9 as compared with that of the control siRNA-treated CIA mice (Fig. 3B). These results indicate that TRAF6 may be involved in the development of CIA.



Figure 4. Anti-TRAF6mAb reduced production of interleukin(IL)-1 $\beta$-induced matrix metalloproteinase (MMP)-1, MMP-3 and MMP-9 by RA-FLSs. FLSs were pretreated with anti-TRAF6mAb for $24 \mathrm{~h}$ and exposed to human IL-1 $\beta$ $(50 \mathrm{ng} / \mathrm{ml})$ for $72 \mathrm{~h}$. MMPs were measured at $570 \mathrm{~nm}$. Data represent the mean \pm standard deviation ${ }^{*} \mathrm{P}<0.01$, vs. control.
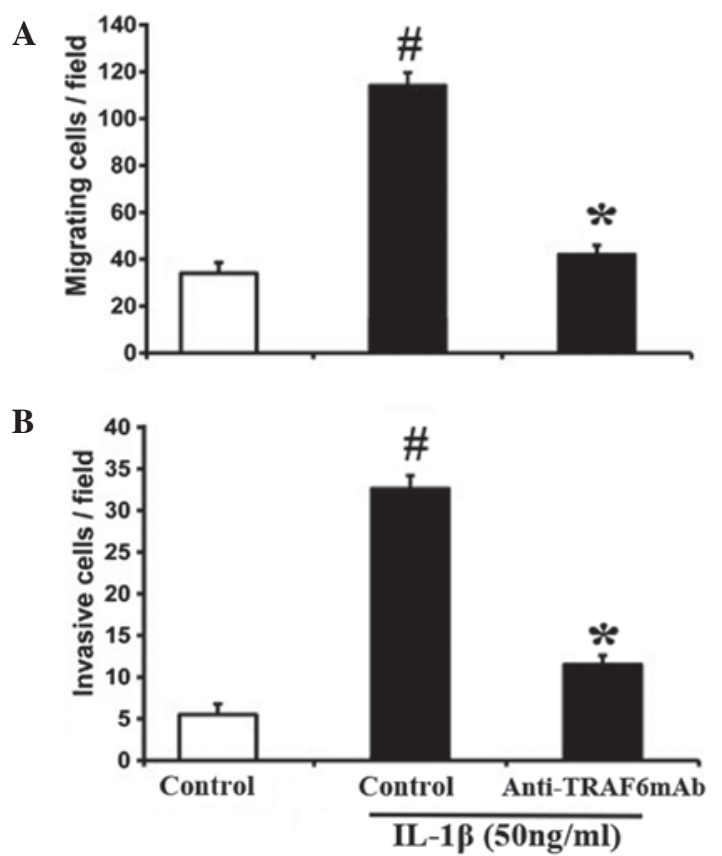

Figure 5. Blockade of TRAF6 inhibited the migration and invasion of rheumatoid arthritis fibroblast-like synoviocytes (RA-FLSs). (A) Anti-TRAF6mAb significantly inhibited the IL-1 $\beta(50 \mathrm{ng} / \mathrm{ml})$-induced migratory behavior of RA-FLSs. (B) Anti-TRAF6mAb significantly reduced the IL-1 $\beta$ $(50 \mathrm{ng} / \mathrm{ml})$-induced invasion of RA-FLSs. Data represent the mean \pm standard deviation ${ }^{\#} \mathrm{P}<0.01$, vs. vehicle-control, $\mathrm{P}<0.01$, vs. control.

Anti-TRAF6mAb reduces the secretion of MMP-1,MMP-3 and MMP-9 by human RA-FLSs. To investigate whether TRAF6 affects the human RA-FLSs in a similar way, the RA-FLSs were pretreated with anti-TRAF6mAb for $24 \mathrm{~h}$, stimulated with human IL-1 $\beta(50 \mathrm{ng} / \mathrm{ml})$ for $72 \mathrm{~h}$ and supernatants were then collected. The results showed that anti-TRAF6mAb reduced the secretion of MMP-1, MMP-3 and MMP-9 by human RA-FLSs in the supernatants as compared with those of the control (Fig. 4). 
Anti-TRAF6mAb restricts the migration of human RA-FLSs. MMPs interact with multiple chemokines and are critically involved in the FLS migration of RA associated with RA progression (14-16). The effect of TRAF6 on the migration and invasion of FLSs was investigated. Treatment with anti-TRAF6mAb markedly inhibited the IL-1 $\beta$-induced migration and invasion of RA-FLSs (Fig. 5). These results indicate that TRAF6 may promote the migratory behavior of human RA-FLSs by potentiating the production of MMPs.

\section{Discussion}

A previous study suggested that FLSs may have the capacity to migrate from joint to joint, potentially explaining the evolution of extensive cartilage and bone deterioration and human RA (17). Recent evidence indicates that the protein TRAF6 is involved in the regulation of autoimmune diseases, including RA and systemic lupus erythmatosus $(18,19)$. However, the role of TRAF6 in RA remains to be investigated. Thus, this study was conducted to explore the potential role of TRAF6 in CIA and human RA-FLSs. In the present study, it was identified that TRAF6 expression was elevated in CIA joints and human RA-FLSs as compared with those in the controls. TRAF6 was knocked down in CIA mice by in vivo siTRAF6. In vivo siTRAF6 treatment reduced the arthritis score and bone destruction. In addition, the production of antibodies and MMPs was reduced by siTRAF6. IL-1 $\beta$-induced RA-FLS migration and invasion were significantly inhibited by anti-TRAF6mAb in vitro. These data suggest that TRAF6 may promote migratory behavior of FLSs in RA. Inhibition of TRAF6 may be a potential therapeutic target for human RA.

TRAF6 has recently been shown to be essential for maintenance of regulatory $\mathrm{T}$ cells $\left(\mathrm{T}_{\text {regs }}\right)$ that suppress $\mathrm{Th} 2$ type autoimmunity (20). Impaired $\mathrm{T}_{\text {reg }}$ function has been shown to be associated with human autoimmune diseases, including RA $(21,22)$. Additionally, TRAF6 has been shown to establish innate immune responses by activating NF- $\mathrm{NB}(23)$ and $\mathrm{NF}-\kappa \mathrm{B}$ activation is widely accepted as a key mediator in the development of RA (24). Previous data have shown that the accumulation of TRAF6-TAK1 complexes and their activation promote osteoclast differentiation (25). TRAF6 is a key mediator in the IL-1 $\beta$-mediated signaling pathway, consequently upregulating its downstream inflammatory events (26). IL-1 $\beta$ is one of the key regulators in the FLS activation in the pathogenesis of RA (27). In the current study, the results revealed that arthritis was attenuated by siTRAF6 in CIA mice, as demonstrated by decreased serum anti-CII, MMP-1, MMP-3 and MMP-9 and reduced histological damage.

Furthermore, the role of TRAF6 in IL-1 $\beta$-induced MMP expression was investigated in RA-FLSs. Blockade of TRAF6 inhibited IL-1 $\beta$-induced production of MMP-1, MMP-3 and MMP-9. These data suggest that TRAF6 may activate FLSs from RA patients, resulting in the elevated expression of MMP-1, MMP-3 and MMP-9. In addition, the data revealed that anti-TRAF6mAb significantly inhibited the IL-1 $\beta$-induced migration and invasion of RA-FLSs.

In conclusion, the results of the present study showed that TRAF6 knockdown reduces the arthritis score and joint destruction via modulating FLS migration and invasion and MMP expression by FLSs. Thus, TRAF6 inhibition may be a potential therapeutic target for RA.

\section{References}

1. Stanford SM, Maestre MF, Campbell AM, Bartok B, Kiosses WB, Boyle DL, Arnett HA, Mustelin T, Firestein GS and Bottini N: Protein tyrosine phosphatase expression profile of rheumatoid arthritis fibroblast-like synoviocytes: a novel role of $\mathrm{SH} 2$ domain-containing phosphatase 2 as a modulator of invasion and survival. Arthritis Rheum 65: 1171-1180, 2013.

2. Kapoor M, Martel-Pelletier J, Lajeunesse D, Pelletier JP and Fahmi H: Role of proinflammatory cytokines in the pathophysiology of osteoarthritis. Nat Rev Rheumatol 7: 33-42, 2011.

3. Paula FS and Alves JD: Non-tumor necrosis factor-based biologic therapies for rheumatoid arthritis: present, future, and insights into pathogenesis. Biologics 8: 1-12, 2014.

4. Inoue J, Gohda $J$ and Akiyama T: Characteristics and biological functions of TRAF6. Adv Exp Med Biol 597: 72-79, 2007.

5. Sun L, Deng L, Ea CK, Xia ZP and Chen ZJ: The TRAF6 ubiquitin ligase and TAK1 kinase mediate IKK activation by BCL10 and MALT1 in T lymphocytes. Mol Cell 14: 289-301, 2004.

6. Chung JY, Lu M, Yin Q, Lin SC and Wu H: Molecular basis for the unique specificity of TRAF6. Adv Exp Med Biol 597: 122-130, 2007.

7. Lee HS, Ka SO, Lee SM, Lee SI, Park JW and Park BH: Overexpression of sirtuin 6 suppresses inflammatory responses and bone destruction in mice with collagen-induced arthritis. Arthritis Rheum 65: 1776-1785, 2013.

8. Nam EJ, Kang JH, Sung S, Sa KH, Kim KH, Seo JS, Kim JH, Han SW, Kim IS and Kang YM: A matrix metalloproteinase 1-cleavable composite peptide derived from transforming growth factor $\beta$-inducible gene h3 potently inhibits collagen-induced arthritis. Arthritis Rheum 65: 1753-1763, 2013.

9. Tarrant TK, Liu P, Rampersad RR, Esserman D, Rothlein LR, Timoshchenko RG, McGinnis MW, Fitzhugh DJ, Patel DD and Fong AM: Decreased Th17 and antigen-specific humoral responses in $\mathrm{CX}_{3} \mathrm{CR} 1$-deficient mice in the collagen- induced arthritis model. Arthritis Rheum 64: 1379-1387, 2012.

10. Nishikawa M, Myoui A, Tomita T, Takahi K, Nampei A and Yoshikawa H: Prevention of the onset and progression of collagen-induced arthritis in rats by the potent p38 mitogen-activated protein kinase inhibitor FR167653. Arthritis Rheum 48: 2670-2681, 2003.

11. Aletaha D, Neogi T, Silman AJ, et al: 2010 Rheumatoid arthritis classification criteria: an American College of rheumatology/european league against rheumatism collaborative initiative. Arthritis Rheum 62: 2569-2581, 2010.

12. Dale J, Purves D, McConnachie A, McInnes I and Porter D: Tightening up? Impact of musculoskeletal ultrasound disease activity assessment on early rheumatoid arthritis patients treated using a treat to target strategy. Arthritis Care Res (Hoboken) 66: 19-26, 2014.

13. Yoshioka Y, Kozawa E, Urakawa H, Arai E, Futamura N, Zhuo L, Kimata K, Ishiguro N and Nishida Y: Suppression of hyaluronan synthesis alleviates inflammatory responses in murine arthritis and in human rheumatoid fibroblasts. Arthritis Rheum 65: 1160-1170, 2013.

14. Lee A, Qiao Y, Grigoriev G, Chen J, Park-Min KH, Park SH, Ivashkiv LB and Kalliolias GD: Tumor necrosis factor $\alpha$ induces sustained signaling and a prolonged and unremitting inflammatory response in rheumatoid arthritis fibroblasts. Arthritis Rheum 65: 928-938, 2013.

15. Laragione T, Brenner M, Sherry B and Gulko PS: CXCL10 and its receptor CXCR3 regulate synovial fibroblast invasion in rheumatoid arthritis. Arthritis Rheum 63: 3274-3283, 2011.

16. García-Vicuña R, Gómez-Gaviro MV, Domínguez-Luis MJ, Pec MK, González-Alvaro I, Alvaro-Gracia JM and Díaz-González F: CC and CXC chemokine receptors mediate migration, proliferation, and matrix metalloproteinase production by fibroblast-like synoviocytes from rheumatoid arthritis patients. Arthritis Rheum 50: 3866-3877, 2004.

17. Cooles FA and Isaacs JD: Pathophysiology of rheumatoid arthritis. Curr Opin Rheumatol 23: 233-240, 2011. 
18. Muto G, Kotani H, Kondo T, Morita R, Tsuruta S, Kobayashi T, Luche H, Fehling HJ, Walsh M, Choi Y and Yoshimura A: TRAF6 is essential for maintenance of regulatory T cells that suppress Th2 type autoimmunity. PLoS One 8: e74639, 2013.

19. Namjou B, Choi CB, Harley IT, Alarcón-Riquelme ME, BIOLUPUS Network, Kelly JA, Glenn SB, et al: Evaluation of TRAF6 in a large multiancestral lupus cohort. Arthritis Rheum 64: 1960-1969, 2012.

20. Muto G, Kotani H, Kondo T, Morita R, Tsuruta S, Kobayashi T, Luche H, Fehling HJ, Walsh M, Choi Y and Yoshimura A: TRAF6 is essential for maintenance of regulatory T cells that suppress Th2 type autoimmunity. PLoS One 8: e74639, 2013.

21. Park JS, Lim MA, Cho ML, Ryu JG, Moon YM, Jhun JY, Byun JK, Kim EK, Hwang SY, Ju JH, Kwok SK and Kim HY: p53 controls autoimmune arthritis via STAT-mediated regulation of the Th17 cell/Treg cell balance in mice. Arthritis Rheum 65: 949-959, 2013.

22. Moon YM, Lee J, Lee SY, Her YM, Ryu JG, Kim EK, Son HJ, Kwok SK, Ju JH, Yang CW, Park SH, Kim HY and Cho ML: Gene-associated retinoid-interferon- induced mortality 19 (GRIM-19) attenuates autoimmune arthritis by regulation of Th17 and Treg cells. Arthritis Rheum 18, 2013.
23. Konno H, Yamamoto T, Yamazaki K, Gohda J, Akiyama T, Semba K, Goto H, Kato A, Yujiri T, Imai T, et al: TRAF6 establishes innate immune responses by activating NF-kappaB and IRF7 upon sensing cytosolic viral RNA and DNA. PLoS One 4: e5674, 2009.

24. Bamborough P, Morse MA and Ray KP: Targeting IKK $\beta$ for the treatment of rheumatoid arthritis. Drug News Perspect 23: 483-490, 2010

25. Wei ZF, Tong B, Xia YF, Lu Q, Chou GX, Wang ZT and Dai Y: Norisoboldine suppresses osteoclast differentiation through preventing the accumulation of TRAF6-TAK1 complexes and activation of MAPKs/NF- $\mathrm{kB} / \mathrm{c}-\mathrm{Fos} / \mathrm{NFATc} 1$ pathways. PLoS One 8: e59171, 2013.

26. Lee HJ, Jang SH, Kim H, Yoon JH and Chung KC: PINK1 stimulates interleukin-1 $\beta$-mediated inflammatory signaling via the positive regulation of TRAF6 and TAK1. Cell Mol Life Sci 69: 3301-3315, 2012.

27. Maruotti N, Grano M, Colucci S, d'Onofrio F and Cantatore FP: Osteoclastogenesis and arthritis. Clin Exp Med 11: 137-145, 2011. 